\title{
Nuclear higher-twist effects in eA DIS
}

\author{
B.G. Zakharov \\ L.D. Landau Institute for Theoretical Physics, GSP-1, 117940, \\ Kosygina Str. 2, 117334 Moscow, Russia
}

\begin{abstract}
We discuss the relation between the treatments of the higher twist nuclear effects in $e A$ DIS based on the pQCD collinear approximation and the light-cone path integral formalism. We show that in the collinear approximation the $N=1$ rescattering contribution to the gluon emission vanishes. It is demonstrated that the nonzero gluon spectrum obtained by Guo, Wang and Zhang is a consequence of unjustified neglect of some terms in the collinear expansion.
\end{abstract}

Keywords: Factorization, Hard processes in nuclei, Collinear approximation

PACS: $12.38 . \mathrm{Bx}, 12.39 . \mathrm{St}, 11.80 . \mathrm{Fv}$

1. In this talk, I focus on the relation between the treatment of the induced gluon emission from the fast struck quark in $e A$ DIS in the higher-twist pQCD approach [1, 2] and in the light-cone path integral (LCPI) approach [3]. The first approach uses the Feynman diagram technique and the collinear approximation (for a review, see [4]). The LCPI formalism is formulated in terms of the wave functions in the coordinate space. At the end of the talk I discuss the physical interpretation of the $1 / Q^{2}$ suppression of soft nuclear rescatterings in jet production without the induced gluon emission.

2. In [1, 2] the twist-4 contribution ( $N=1$ rescattering) to the gluon spectrum has been expressed in terms of the diagrams like shown in Fig. 1. The lower soft parts of the
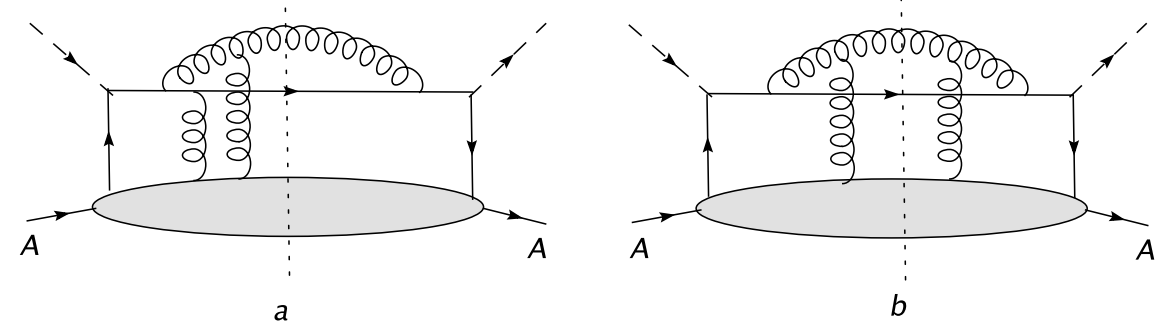

FIGURE 1. Examples of the diagrams for gluon emission in the higher-twist approach [1, 2].

diagrams are expressed via the matrix element $\left\langle A\left|\bar{\psi}(0) A^{+}\left(y_{1}\right) A^{+}\left(y_{2}\right) \psi\left(y_{3}\right)\right| A\right\rangle$. The upper hard parts, $H$, are calculated perturbatively. The contour integrations over the $p^{+}$ momentum components are carried out with the help of the poles in the denominators of the retarded propagators for fast quarks and gluon

$$
G_{r e t}(y)=\frac{i}{(2 \pi)^{4}} \int_{p^{-}>0} d p^{+} d p^{-} d \vec{p}_{T} \frac{\exp \left[-i\left(p^{+} y^{-}+p^{-} y^{+}-\vec{p}_{T} \vec{y}_{T}\right)\right]}{2 p^{-}\left(p^{+}-\frac{\vec{p}_{T}^{2}+m^{2}-i 0}{2 p^{-}}\right)}
$$

$\left(p^{ \pm}=\left(p^{0} \pm p^{3}\right) / \sqrt{2}\right.$, the virtual photon momentum is chosen in the negative $z$ direction, for simplicity spin is ignored). The collinear approximation corresponds to replacement 
of the hard part by its second order expansion in the $t$-channel transverse gluon momentum $\vec{k}_{T}$ (only the second order term is important)

$$
H\left(\vec{k}_{T}\right) \approx H\left(\vec{k}_{T}=0\right)+\left.\frac{\partial H}{\partial k_{T}^{\alpha}}\right|_{\vec{k}_{T}=0} k_{T}^{\alpha}+\left.\frac{\partial^{2} H}{\partial k_{T}^{\alpha} \partial k_{T}^{\beta}}\right|_{\vec{k}_{T}=0} \cdot \frac{k_{T}^{\alpha} k_{T}^{\beta}}{2} .
$$

3. In the LCPI approach the amplitude for $a \rightarrow b c$ partonic process is written in terms of the incoming $(i=a)$ and outgoing $(i=b, c)$ wave functions. These functions are written as $\psi_{i}(y)=\frac{1}{\sqrt{2 p_{i}^{-}}} \exp \left[-i p_{i}^{-} y^{+}\right] \phi_{i}\left(y^{-}, \vec{y}_{T}\right)$. The $y^{-}$dependence of the transverse wave functions $\phi_{i}$ is governed by the two-dimensional Schrödinger equation

$$
i \frac{\partial \phi_{i}\left(y^{-}, \vec{y}_{T}\right)}{\partial y^{-}}=\left\{\frac{\left[\left(\vec{p}_{T}-g \vec{A}_{T}\right)^{2}+m_{i}^{2}\right]}{2 p_{i}^{-}}+g A^{+}\right\} \phi_{i}\left(y^{-}, \vec{y}_{T}\right) .
$$

Diagrammatically the gluon emission from the struck quark can be described by the graph shown in Fig. 2, where the horizontal lines show the Green's functions $\mathscr{K}(\rightarrow)$ and $\mathscr{K}^{*}(\leftarrow)$ of the equation (3). The integration over $y^{+}$of the frequently oscillating factors

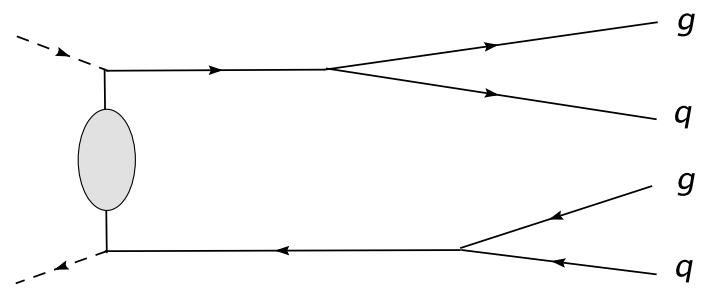

FIGURE 2. Schematic diagram for gluon emission in the LCPI approach [3].

can be done in the same way as for the ordinary quark distribution $f_{q}$. As compared to the Collins-Soper formula $f_{q}=\frac{1}{4 \pi} \int d y^{-} e^{i x_{B} P^{+} y^{-}}\left\langle N\left|\bar{\psi}\left(-y^{-} / 2\right) \gamma^{+} \psi\left(y^{-} / 2\right)\right| N\right\rangle$, now $e^{i x_{B} P^{+} y^{-}}$is replaced by

$e^{i x_{B} P^{+} y^{-}} \int d \xi d \xi^{\prime} \mathscr{K}_{q_{f}}(\infty \mid \xi) \mathscr{K}_{g}(\infty \mid \xi) \mathscr{K}_{q_{i}}\left(\xi \mid y^{-} / 2\right) \mathscr{K}_{q_{f}}^{*}\left(\infty \mid \xi^{\prime}\right) \mathscr{K}_{g}^{*}\left(\infty \mid \xi^{\prime}\right) \mathscr{K}_{q_{i}}^{*}\left(\xi^{\prime} \mid-y^{-} / 2\right)$

(for simplicity we omit all the transverse coordinates). One can show that the LCPI formalism can be obtained from the pQCD treatment in terms of Feynman diagrams. Indeed, the retarded propagator (1) can be written in terms of the zeroth-order (in $A_{\mu}$ ) Green's function of the Schrödinger equation (3) (we denote it $K$ ) as

$$
G_{r e t}(y)=\int_{0}^{\infty} \frac{d k^{-}}{4 \pi k^{-}} e^{-i k^{-} y^{+}} K\left(\vec{y}_{T}, y^{-} \mid 0,0\right) .
$$

The integration in the Feynman diagrams over $y^{+}$gives conservations of the large $k^{-}$ components in each vertex, and we reproduce exactly the LCPI expression in terms of the transverse Green's functions.

4. The typical difference in the coordinate $y^{-}$for the upper and lower $\gamma^{*} q q$ vertices in Fig. 2 (which gives the scale of the quantum nonlocality of the fast quark production) 
is given by the well known Ioffe length $L_{I}=1 / m_{N} x_{B}$. For moderate values of $x_{B}$ this scale is much smaller than the typical scale of the gluon emission. It allows one to treat the quark production and gluon emission as mutually independent. Then for a given position $\mathbf{r}$ of the struck nucleon the generalized quark distribution as a function of the gluon fractional momentum $z$ can be approximated by the factorized form

$$
d f_{q}(z, \mathbf{r}) / d z \approx f_{q} d P(z, \mathbf{r}) / d z
$$

where the quark distribution $f_{q}$ stems from the left part of the diagram in Fig. 2, and the gluon spectrum $d P / d z$ is described by the right parts of the diagram evaluated neglecting the quantum nonlocality of the fast quark production.

In the LCPI approach [3] the final formula for $d P / d z$ is expressed in terms of the Green's function for the two-dimensional Schrödinger equation with an imaginary potential which is proportional to the dipole cross section $\sigma(\rho)$. The Hamiltonian takes the harmonic oscillator form for a quadratic parametrization $\sigma(\rho)=C \rho^{2}$. The $N=1$ contribution to the gluon spectrum in the oscillator approximation should coincide with prediction of the collinear approximation in the treatment [1, 2]. Indeed, the quadratic form of the dipole cross section corresponds to the vector potential approximated by the linear expansion $A^{+}\left(y^{-}, \vec{y}_{T}+\vec{\rho}\right) \approx A^{+}\left(y^{-}, \vec{y}_{T}\right)+\vec{\rho} \nabla_{y_{T}} A^{+}\left(y^{-}, \vec{y}_{T}\right)$ which can be traced back to the collinear expansion in the momentum space. For massless partons in the oscillator approximation the spectrum of the LCPI approach coincides with the spectrum in the BDMPS formalism [5]. For a target of thickness $L$ it reads [5, 6]

$$
\frac{d P}{d z}=\frac{\alpha_{s} P_{G q}(z)}{\pi} \ln |\cos \Omega L|,
$$

where $P_{G q}$ is the ordinary splitting function, $\Omega=\sqrt{-i C_{3} n / z(1-z) E}, C_{3}=C C_{A} / C_{F}$. The expansion of the spectrum (6) in the density starts with the terms $\propto n^{2}$ which corresponds to the $N=2$ rescatterings [6], and the contribution of $N=1$ rescattering is absent. The fact that the $N=1$ gluon spectrum vanishes in the collinear approximation can also be demonstrated by the direct calculations of the second derivative of the hard part in the momentum space [6, 7].

5. Let us now discuss why a nonzero $N=1$ spectrum has been obtained in [1, 2]. In [1, 2] the nonzero second derivative of the hard part (at $z \ll 1)$ comes from the graph shown in Fig. $1 \mathrm{~b}$ (at $z \ll 1$ ). The authors use for the integration variable in the hard part of this graph the transverse momentum of the final gluon, $\vec{l}_{T}$. The $\vec{l}_{T}$-integrated hard part obtained in [2] (Eq. 15 of [2]) reads (up to an unimportant factor)

$$
\begin{gathered}
H\left(\vec{k}_{T}\right) \propto \int \frac{d \vec{l}_{T}}{\left(\vec{l}_{T}-\vec{k}_{T}\right)^{2}} R\left(y^{-}, y_{1}^{-}, y_{2}^{-}, \vec{l}_{T}, \vec{k}_{T}\right), \\
R\left(y^{-}, y_{1}^{-}, y_{2}^{-}, \vec{l}_{T}, \vec{k}_{T}\right)=\frac{1}{2} \exp \left[i \frac{y^{-}\left(\vec{l}_{T}-\vec{k}_{T}\right)^{2}-(1-z)\left(y_{1}^{-}-y_{2}^{-}\right)\left(\vec{k}_{T}^{2}-2 \vec{l}_{T} \vec{k}_{T}\right)}{2 q^{-} z(1-z)}\right] \\
\times\left[1-\exp \left(i \frac{\left(y_{1}^{-}-y^{-}\right)\left(\vec{l}_{T}-\vec{k}_{T}\right)^{2}}{2 q^{-} z(1-z)}\right)\right] \cdot\left[1-\exp \left(-i \frac{y_{2}^{-}\left(\vec{l}_{T}-\vec{k}_{T}\right)^{2}}{2 q^{-} z(1-z)}\right)\right]
\end{gathered}
$$


$\left(y^{-}, y_{1,2}^{-}\right.$correspond to the quark interactions with the virtual photon and $t$-channel gluons, our $z$ equals $1-z$ in [1, 2]). The dominating nuclear-size enhanced configurations correspond to $\left|y_{1}^{-}-y_{2}^{-}\right| \lesssim R_{N},\left|y^{-}\right| \lesssim R_{N}\left(R_{N}\right.$ is the nucleon size). This contribution can be calculated taking $y_{1}^{-}=y_{2}^{-}$. A simple calculation gives

$$
\left.\nabla_{k_{T}}^{2} H\right|_{\vec{k}_{T}=0} \propto 4 \pi \int_{0}^{\infty} d l_{T}^{2}\left\{\frac{1-\cos \left(a l_{T}^{2}\right)}{l_{T}^{4}}-\frac{a \sin \left(a l_{T}^{2}\right)}{l_{T}^{2}}+a^{2} \cos \left(a l_{T}^{2}\right)\right\},
$$

where $a=y_{1} / 2 q^{-} z(1-z)$. It gives $\left.\nabla_{k_{T}}^{2} H\right|_{\vec{k}_{T}=0}=0$. Indeed, after integrating the first term by parts it cancels the contribution from the second term in (9). The last integral equals zero. The last two terms in the integrand in (9) which come from differentiating the factor $R$ have been unjustifiedly neglected in [1, 2]. It is for this reason that the authors have obtained the nonzero spectrum.

6. The pQCD calculations within the collinear approximation [4] show that the $N=1$ rescattering contribution in jet production (in $e A$ DIS or in $h A$ collisions) is $\propto A^{1 / 3} \times$ $\lambda / Q^{2}$, where $\lambda$ can be expressed in terms the higher-twist parton distribution. The fact that the additional soft rescattering is suppressed by $1 / Q^{2}$ is surprising. In [4] this fact was attributed to the off-shell suppression of the rescattering cross section for virtual partons. However, this interpretation is inconsistent with the uncertainty relation $\Delta E \Delta t \gtrsim 1$. Let us consider jet production in $h A$-collisions. In this case from the uncertainty relation one can obtain for the $L$-dependence of the fast parton virtuality $Q^{2}(L) \sim Q_{i n}^{2} / L m$ ( $m \sim m_{N} / 3, Q_{i n} \sim p$ ). It says that the typical virtuality at $L \sim R_{A}$ (which dominates the soft rescatterings) becomes small $\left\langle 1 / Q^{2}\right\rangle \gg 1 / Q_{i n}^{2}$. Note also that in pQCD calculations the distance between the parton production point and rescattering does not appear in the formula for rescattering contribution at all. It makes clear that $1 / Q^{2}$ suppression is not due to the parton off-shellness. A simple interpretation of the $1 / Q^{2}$ suppression can be given in the LCPI approach. Jet production in a large nucleus at the amplitude level can be described by the diagram shown in Fig. 3a, and the cross section is described by
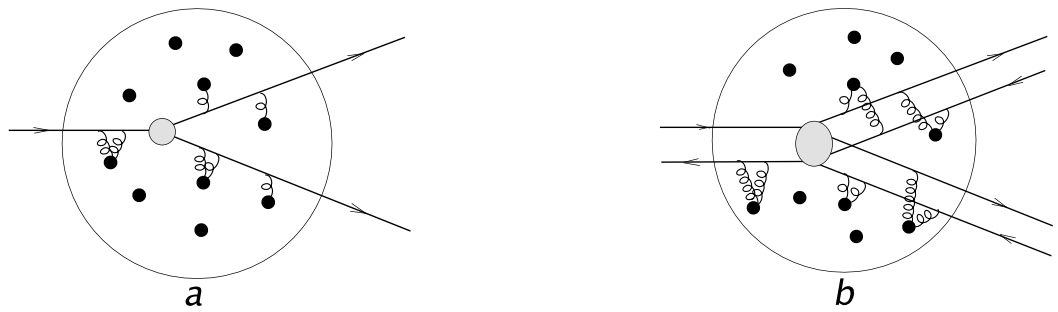

FIGURE 3. Diagrams for the amplitude (a) and cross section (b) of jet production on a nucleus.

the diagram shown in Fig. 3b. For each nucleon the sum of the double gluon exchange diagrams which appear in Fig. $3 b$ can be written in terms of the dipole cross section $\sigma(\rho)$. The in-medium evolution of the "parton-antiparton" systems in Fig. $3 b$ are described by the evolution operator for the density matrix which can be calculated exactly in the path integral method [8, 3]. Remarkably, the path integral calculation reproduces the result of the eikonal approximation which neglects the transverse motion. It means that the trajectories in Fig. $3 \mathrm{~b}$ are essentially the straight lines. Since the transverse distance between $\rightarrow$ and $\leftarrow$ lines is $\sim 1 / p$ using $\sigma(\rho) \sim \alpha_{s}^{2} \rho^{2} x f_{g}(x, p \sim 1 / \rho)$, we obtain 
for the nuclear correction $\delta \sigma_{A}^{\text {hard }}(p) / \sigma_{N}^{\text {hard }}(p) \sim n_{A} L x f_{g}(x, p) / p^{2}$ (here $f_{g}$ is the gluon density, $x \sim 0)$. This result can be reproduced in the momentum space as well. The $p_{T}$ broadening distribution for each parton reads [8, 3]

$$
I(\vec{q})=\frac{1}{(2 \pi)^{2}} \int d \vec{\rho} \exp [i \vec{q} \vec{\rho}-L \sigma(\rho) n / 2]
$$

This $I(\vec{q})$ coincides with the prediction of the classical transport equation evaluated with rescattering cross section $d \sigma / d q^{2}$ connected with $\sigma(\rho)$ by the relation $\sigma(\rho)=$ $\frac{2}{\pi} \int d \vec{q}[1-\exp (i \vec{q} \vec{\rho})] \frac{d \sigma}{d q^{2}}$. The in-medium hard cross section has a simple form

$$
\sigma_{A}^{\text {hard }}(\vec{p})=\int d \vec{q} \sigma_{N}^{h a r d}(\vec{p}-\vec{q}) I(\vec{q}) \approx \sigma_{N}^{\text {hard }}(\vec{p})+\frac{1}{4}\left(\frac{\partial}{\partial \vec{p}}\right)^{2} \sigma_{N}^{\text {hard }}(\vec{p}) \int_{p>q} d \vec{q} q^{2} I(\vec{q})
$$

which gives for the $N=1$ rescattering correction $\delta \sigma_{A}^{\text {hard }}(p) / \sigma_{N}^{\text {hard }}(p) \sim\left\langle q^{2}(p)\right\rangle / p^{2}$.

One remark on the form of $I(\mathbf{q})$ is in order here. The collinear expansion gives Gaussian $I(\mathbf{q})$ [9]. However, one can easily show that for real nuclei the $I(\mathbf{q})$ calculated accounting for the Coulomb effects turns out to be essentially non-Gaussian due to smallness of the nucleus size.

7. In summary, we have demonstrated that the collinear expansion gives a zero $N=$ 1 rescattering contribution to the gluon spectrum in $e A$ DIS. The nonzero spectrum obtained in [1, 2] is a consequence of unjustified neglect of some terms in the collinear expansion. The $1 / Q^{2}$ suppression of soft nuclear rescatterings is not an off-shell effect. The effect has a simple classical interpretation. The collinear approximation can not be used for evaluation of the form of the $p_{T}$-broadening distribition for real nuclei.

\section{ACKNOWLEDGMENTS}

I am grateful to R. Fiore and J. Soffer for their hospitality at Diffraction 2008. I also am thankful to P. Aurenche and H. Zaraket for collaboration. This work is supported in part by the grant RFBR 06-02-16078-a and the ENS-Landau program.

\section{REFERENCES}

1. X.N. Wang and X. Guo, Nucl. Phys. A696 (2001) 788.

2. B.W. Zhang and X.N. Wang, Nucl. Phys. A720 (2003) 429.

3. B.G. Zakharov, JETP Lett. 63 (1996) 952; ibid. 65, (1997) 615; 70 (1999) 176; Phys. Atom. Nucl. 61 (1998) 838.

4. J. Qiu and G. Sterman, Int. J. Mod. Phys. E12 (2003) 149.

5. R. Baier, Y.L. Dokshitzer, A.H. Mueller, S. Peigné, and D. Schiff, Nucl. Phys. B483, 291 (1997); ibid. B484, 265 (1997).

6. B.G. Zakharov, JETP Lett. 73 (2001) 49.

7. P. Aurenche, B.G. Zakharov, and H. Zaraket, JETP Lett. 87, 605 (2008) [arXiv:0804.4282 [hep-ph]].

8. B.G. Zakharov, Sov. J. Nucl. Phys. 46 (1987) 92.

9. A. Majumder and B. Mueller, Phys. Rev. C77 (2008) 054903. 